\title{
Performance Comparison of PM Synchronous and PM Vernier Machines Based on Equal Output Power per Unit Volume
}

\author{
Dae-Kyu Jang* and Jung-Hwan Chang ${ }^{\dagger}$
}

\begin{abstract}
This paper compares the performances of permanent-magnet synchronous (PMS) and permanent-magnet vernier (PMV) machines for low-speed and high-torque applications. For comparison with the PMS machines, we consider two types of the PMV machine. The first one has surface-mounted permanent magnets (PMs) on the rotor and the other has PMs inserted on both sides of the stator and rotor. The PMS and PMV machines are designed to meet the condition of equal output power per unit volume. We analyze the magnetic fields of the machines using a twodimensional finite element analysis (FEA). We then compare their performances in terms of the generated torque characteristics, power factor, loss, and efficiency.
\end{abstract}

Keywords: Direct-drive, Efficiency, PM synchronous machine, PM vernier machine, Output power

\section{Introduction}

In low-speed direct-drive applications, a permanent magnet synchronous (PMS) machine often has a high number of poles. As the number of poles increases, the thickness of the stator and rotor yokes can be made thin because of the low flux per pole. Therefore, in the limited external diameter of the machine, the air-gap diameter can be maximized, leading to high torque. However, PMS machines with a high number of poles require a high number of slots to enable normal operation. Unlike PMS machines, permanent magnet vernier (PMV) machines can operate with different combinations of large number of poles and small number of slots because of their magnetic gearing effect. PMV machines are commonly known as high-torque machines. In recent years, studies of PMV machines have focused on their topologies in order to improve their performance parameters such as the torque density and power factor [1-5]. However, compared with PMS machines in the same conditions, the distinct characteristics and advantages of the magnetic gearing effect for high-torque applications have not yet been reported. This paper aims to investigate the influence of the magnetic gearing effect on direct-drive applications by comparing the performances of PMS and PMV machines that have a similarly high number of poles.

Two types of PMV machines have been considered for comparison with the PMS machine. One is the surfacemounted PM type, which has flux-modulation poles (FMPs) on the stator and PMs on the surface of the rotor $[1,2]$. In the other type, the PMs are inserted between the FMPs on the stator and the teeth on the rotor [3]. For a

$\dagger$ Corresponding Author: Department of Electrical Engineering, Donga University, Busan, Korea. (cjhwan@dau.ac.kr)

* Department of Electrical Engineering, Donga University, Busan, Korea. (cjhwan@dau.ac.kr)

Received: May 8, 2015; Accepted: September 30, 2015 fair comparison, we designed the PMS and two types of PMV machines based on the constraint conditions of identical output power and size. This was done using a two-dimensional (2-D) finite element analysis (FEA) method. Then, we compared their performances in terms of the generated torque characteristics, power factor, loss, and efficiency. In PMV machines, the magnetic gearing effect reduces the number of slots and the number of turns per phase when compared with the PMS machine. In addition, the PMV machines generate the same output power as the PMS machine by using a smaller amount of the PM material compared to that of the PMS machine. Although the PMV machines have low power factor values, the analysis results for the efficiency characteristics show the potential applicability of PMV machines in direct-drive applications.

\section{PMS and PMV Machines}

\subsection{Structure}

Fig. 1 shows a cross-section view of a PMS machine with a high number of poles for both low-speed and hightorque applications. The stator has 36 slots and three phaseconcentrated windings to generate the rotating magnetic field of 12 pole pairs. The external rotor has 48 magnetic poles by surface-mounted permanent magnets (PMs). In order to obtain the sinusoidal waveform of the back electromagnetic force (EMF), we designed the stator teeth using the smaller radius, $R_{t}$ of curvature of the teeth instead of the outer radius, $R_{o}$ of the stator.

In the PMV machines, the magnetic field caused by the stator is modulated, and corresponds to the magnetic poles of the rotor by the FMPs when the following relationship is satisfied [4]. 


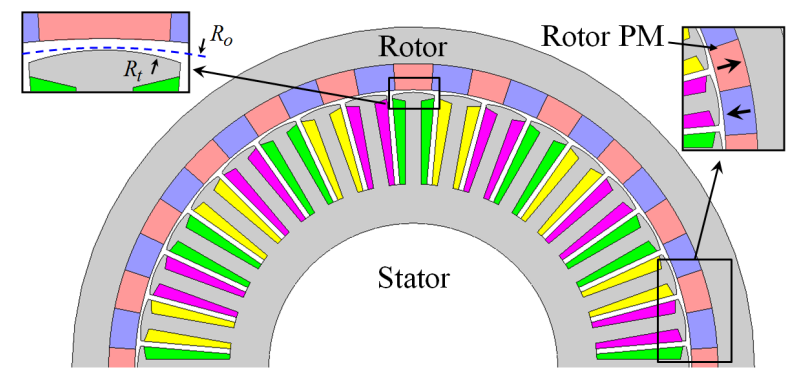

Fig. 1. Structure of a PMS machine

$$
P_{r}=N_{s}-P_{s}
$$

where $P_{\mathrm{r}}, P_{\mathrm{s}}$, and $N_{\mathrm{s}}$ are the number of rotor pole pairs, stator pole pairs, and FMPs, respectively. Moreover, this phenomenon, which is called the magnetic gearing effect, reduces the rotational speed of the modulated armature field. Thus, the rotor is synchronized with the modulated armature field and rotates at a speed that is lower than that of the armature field as follows.

$$
G_{r}=\frac{\left|P_{s}-N_{s}\right|}{P_{s}}=\left|\frac{\omega_{s}}{\omega_{r}}\right|
$$

where $G_{r}$ is the speed-reduction ratio and $\omega_{s}$ and $\omega_{r}$ are the rotational speeds of the fundamental field of the armature and the outer rotor, respectively.

Fig. 2 shows the cross section of PMV machines I and II. The stators of the PMV machines have six slots and three phase windings for the rotating magnetic field of the two pole pairs. Each tooth is split into four FMPs. According to (1) and (2), the number of rotor pole pairs and the speed-

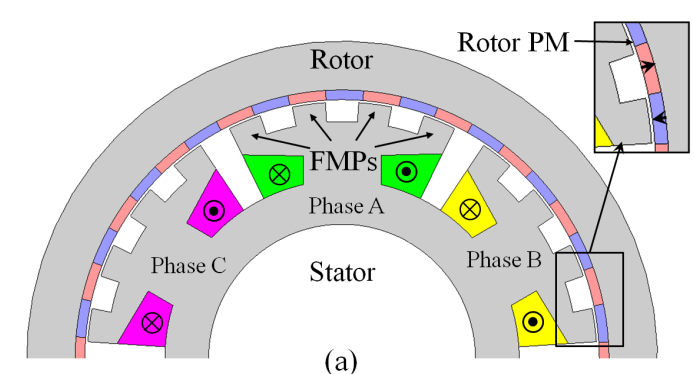

(a)

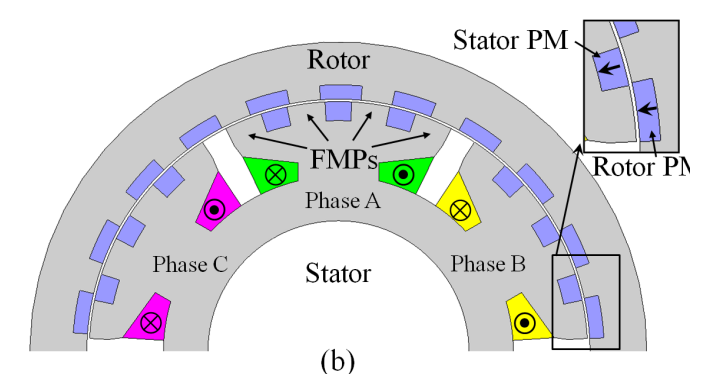

(b)

Fig. 2. Structure of the PMV machines: (a) PMV machine I; (b) PMV machine II reduction ratio are set to 22 and 11 , respectively. In the case of PMV machine I, the rotor has the surface-mounted PMs. PMV machine II has PMs embedded on both sides of the stator and rotor, and all of the PMs are magnetized in the inward radial direction.

\subsection{Specification}

For a fair comparison, we designed the PMS and PMV machines such that they generate an equal output power with the conditions being that they have the same size and comprise the same magnetic materials. The basic dimensions of the machines are as follows. The outer diameter of the rotor is $260 \mathrm{~mm}$, the outer diameter of the stator is $210 \mathrm{~mm}$, the air-gap length is $1 \mathrm{~mm}$, and the core lamination is 26 $\mathrm{mm}$. In addition, the core materials are 35PN440 and the $\mathrm{PMs}$ are NdFeB magnets that have a residual flux density of $1.2 \mathrm{~T}$.

When designing the machines, the thickness of the rotor PM and the number of turns per phase are critical variables for determining the amplitude of the generated torque. In particular, depending on the thickness of the rotor PM, the PMS and PMV machines have different torque characteristics that are due to the magnetic saturations in the rotor yoke. First, the number of turns per phase for the PMS and PMV machines are set to have an identical back EMF when the thickness of the rotor PM is $10 \mathrm{~mm}$. For an input current of $2 \mathrm{~A}$, we used the 2-D FEA commercial software FLUX 2-D to analyze the torque characteristics in order to adjust the PM thickness and the number of turns. Fig. 3 shows the variations of the generated torque in the PMS and PMV machines according to the thickness of the rotor PM. The thickness of the rotor PM was changed by adjusting the thickness of the rotor yoke when the inner and outer diameters of the rotor were fixed. The average torque for the PMS machine and PMV machine is maximum for a rotor PM thickness of 10 and $4 \mathrm{~mm}$, respectively. In addition, the torque ripples of the PMS machine and PMV machine I are less than 11\% although the values of the torque ripple tend to increase with an increasing rotor PM thickness. Thus, in terms of the maximum torque, the optimum thickness of the rotor PM in the PMS machine and PMV machine I can be determined to be 10 and $4 \mathrm{~mm}$, respectively. In the case of PMV machine II, the thickness of the rotor PM for the maximum generated torque is $8 \mathrm{~mm}$. However, when the thickness of the rotor PM was changed from 6 to $8 \mathrm{~mm}$, the average torque improved only by $0.6 \%$, whereas the torque ripple increased by $17 \%$. Therefore, the optimum thickness of the rotor PM in the PMV machine II is set to $6 \mathrm{~mm}$. After determining the optimum thickness of the rotor PM, the numbers of turns per phase for PMV machines is adjusted to generate the same torque with the PMS machine.

Table 1 shows the winding specifications of the PMS and PMV machines. The number of turns per phase are 


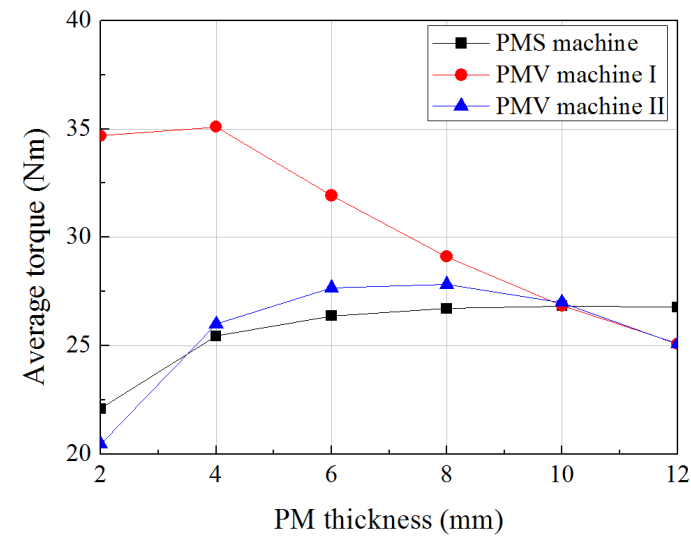

(a)

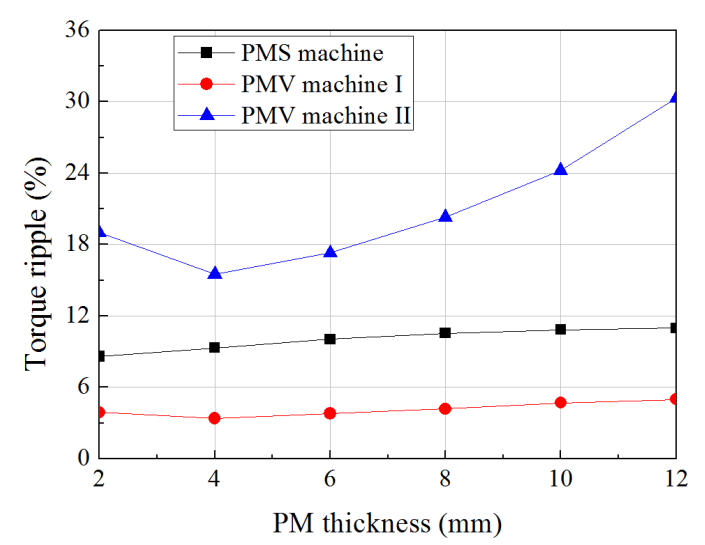

(b)

Fig. 3. Variation of the generated torque according to the thickness of the rotor PM in the PMS and PMV machines: (a) Average torque; (b) Torque ripple

Table 1. Winding specifications

\begin{tabular}{|c|c|c|c|c|}
\hline & & PMS & PMV I & PMV II \\
\hline \multicolumn{2}{|c|}{ Number of turns per phase } & 1200 & 562 & 322 \\
\hline \multicolumn{2}{|c|}{ Coil fill factor [\%] } & 57 & 57 & 57 \\
\hline \multicolumn{2}{|c|}{ Coil diameter $[\mathrm{mm}]$} & 0.91 & 0.91 & 1.15 \\
\hline \multirow{2}{*}{ Resistance $[\Omega]$} & End winding & 1.28 & 3.13 & 1.79 \\
\hline & Slot area & 1.69 & 0.80 & 0.45 \\
\hline
\end{tabular}

1200, 562, and 322 respectively for the PMS machine, PMV machine I, and PMV machine II, respectively. For each machine, the diameter of the coil and the area of the slot are determined for an identical fill factor of $57 \%$. The diameter of the coils for the PMS machine and PMV machine $\mathrm{I}$ is $0.91 \mathrm{~mm}$ (AWG 19), and the diameter of the coils for the PMV machines II is $1.15 \mathrm{~mm}$ (AWG 17). Compared with the PMS machine, the number of turns for each phase of PMV machines I and II are reduced by 53\% and $73 \%$, respectively. However, the width of the teeth and the number of turns per slot are increased, and this causes an increase in the resistance in the end windings, as shown in Table 1.

\section{Performance Comparison}

We calculated the magnetic fields of the PMS and PMV machines when an input current of $2 \mathrm{~A}$ is fed into the coils and the machines operate at $150 \mathrm{rpm}$. Fig. 4 shows the distribution of the radial component of the flux density in the air-gap region. For all of the machines, the harmonic order with the highest amplitude corresponds to the number of rotor pole pairs. In the PMV machines, the 2nd harmonic is caused by the armature winding, and it is modulated into the 22nd harmonics because of the magnetic gearing effect. In addition, the 24th harmonic in PMV machine II is generated by the magnetic interaction between the the stator PMs and FMPs. For the PMS

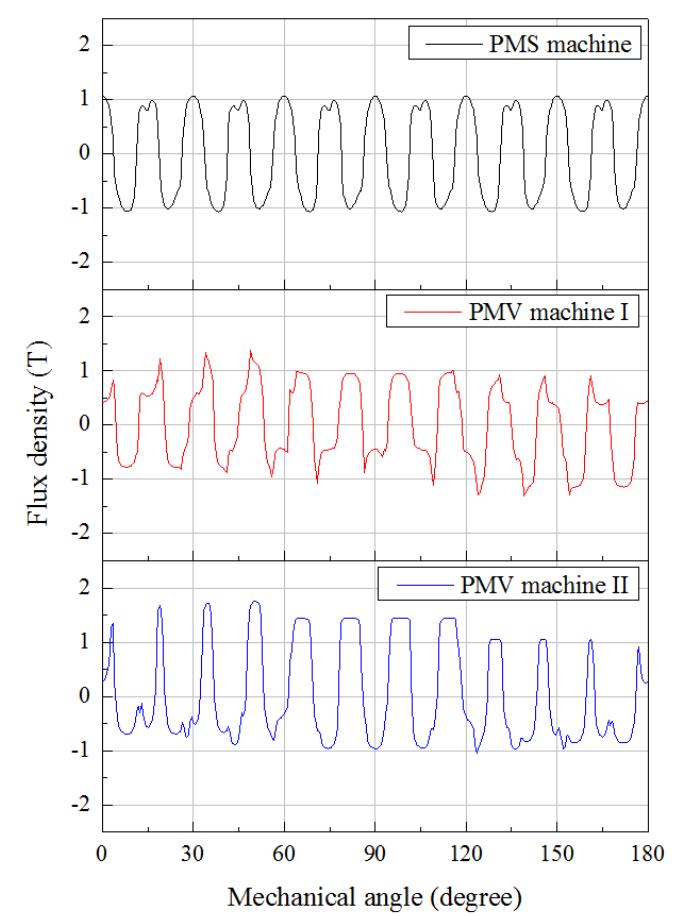

(a)

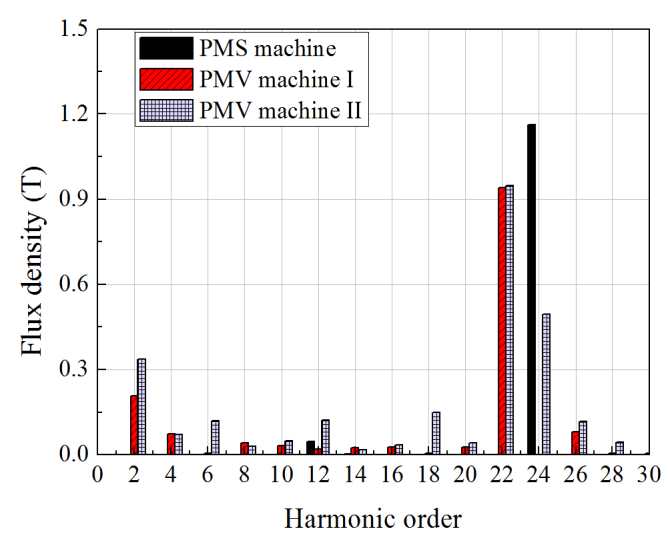

(b)

Fig. 4. Distribution of the radial component of the flux density in the air gap (a) Waveforms (b) FFT results 
machine, the frequency of the input current is $60 \mathrm{~Hz}$, which synchronizes with the 48 poles of the rotor to rotate at 150 rpm. The frequency of the PMV machines is $55 \mathrm{~Hz}$ for a rotor speed of $150 \mathrm{rpm}$.

\subsection{Output power}

Fig. 5 shows the waveforms of the torque generated in the PMS and PMV machines. The average torque generated in each machine has the same value of $27 \mathrm{Nm}$, which indicates that the machines have an equal output power per unit volume of the machine. However, compared with the PMS machine, the volume of the PMs in PMV machines I and II are reduced by $61 \%$ and $44 \%$, respectively. The output power per unit volume of the PMs are $2.32,5.98$, and $4.19 \mathrm{~kW} / \mathrm{cm}^{3}$, repectively for the PMS machine, PMV machine I, and PMV machine II. The PMV machine I achieves the smallest torque ripple of $3.6 \%$. On the contrary, the torque ripple generated in PMV machine II has the highest value of $19.8 \%$, which is 1.8 times greater than that of the PMS machine.

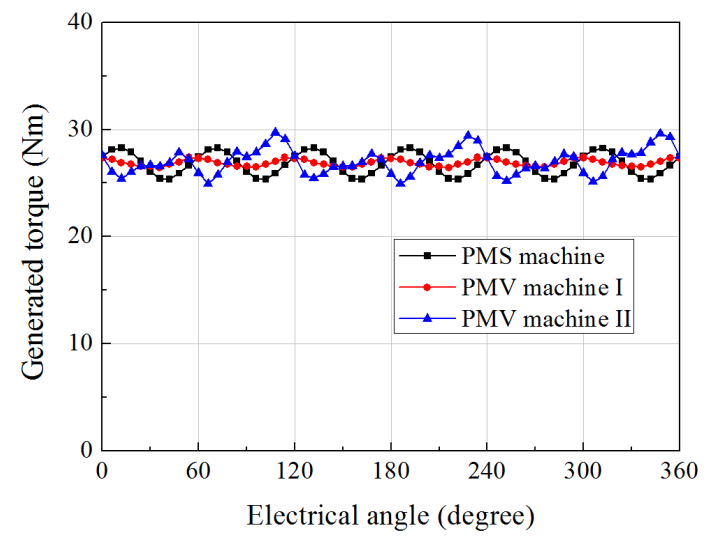

Fig. 5. The waveforms of the generated torque

\subsection{Power factor}

The power factor is defined as the ratio of the real power to apparent power. If the waveforms of the terminal voltage and input current are assumed to be sinusoidal, the power factor is obtained by the cosine values at the phase angles between the terminal voltage and the input current. In the FEA with the current source, the terminal voltage can be calculated by the voltage equations as follows.

$$
v_{a}=i_{a} R_{a}+L_{s} \frac{d i_{a}}{d t}+e_{a}
$$

The terminal voltage, $v_{a}$ consists of three parts. These are the armature-resistance voltage drop, $i_{a} R_{a}$, the inductorinduced voltage of the stator winding, $L_{s} \cdot d i_{a} / d t$, and the back EMF at no-load, $e_{a}$.
Table 2. The components of the terminal voltage in the PMS and PMV machines

\begin{tabular}{c|c|c|c}
\hline & PMS & PMV I & PMV II \\
\hline$i_{a} \cdot R_{a}\left[\mathrm{~V}_{\mathrm{rms}}\right]$ & 5.93 & 7.85 & 4.47 \\
\hline$L_{s} \cdot d i_{a} / d t\left[\mathrm{~V}_{\mathrm{rms}}\right]$ & 29.58 & 117.01 & 57.94 \\
\hline$e_{a}\left[\mathrm{~V}_{\mathrm{rms}}\right]$ & 100.85 & 99.49 & 102.14 \\
\hline$v_{a}\left[\mathrm{~V}_{\mathrm{rms}}\right]$ & 110.81 & 158.79 & 121.34 \\
\hline
\end{tabular}

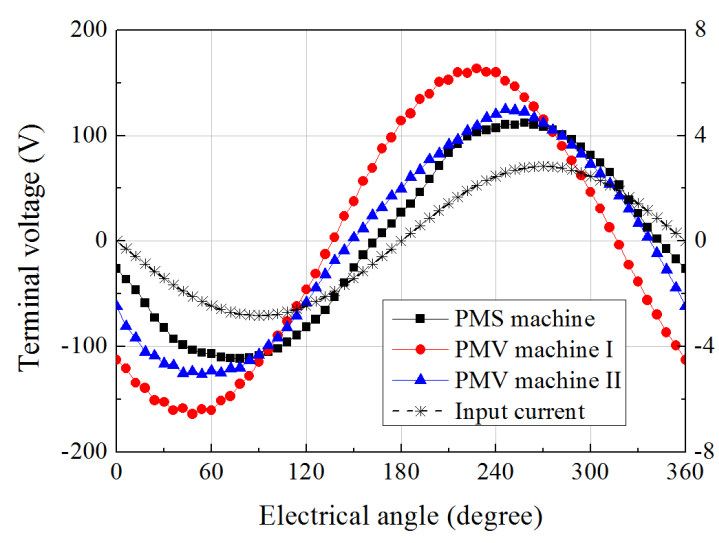

Fig. 6. The waveforms of the terminal voltage

Table 2 shows each component of the terminal voltages for the PMS and PMV machines. The back EMF for each of the machines has a similar value within the range of $3 \%$. In addition, the voltage drops in the armature resistances have very low values relative to the back EMF. On the contrary, the induced voltages in the inductors of the PMV machines have much higher values than that of the PMS machine. This results in a decrease of the power factor in the PMV machines. Fig. 6 shows the waveforms of the terminal voltage for the PMS and PMV machines. The phase angles between the terminal voltage and the input current are $15.48^{\circ}, 47.47^{\circ}$, and $28.52^{\circ}$, for the PMS machine, PMV machine I, and PMV machine II, respectively. The power factors of the PMS machine, PMV machine I, and PMV machine II are 0.96, 0.68, and 0.88, respectively. This indicates that PMV machines require power factor-correction methods, which increases the cost of their control systems.

\subsection{Efficiency}

Table 3 compares the efficiency of the PMS and PMV machines, which is obtained using the following equation:

$$
\eta=\frac{P_{\text {out }}}{P_{\text {out }}+P_{\text {copper }}+P_{\text {iron }}}
$$

where $P_{\text {out }}, P_{\text {copper }}$, and $P_{\text {iron }}$ are the output power, copper loss, and iron loss, respectively. The iron loss is calculated based on the iron loss model proposed by Bertotti [6]. Compared with the PMS machine, the efficiency of PMV machine $\mathrm{I}$ is decreased by $2 \%$ and the efficiency of PMV 
Table 3. The components of the terminal voltage for the PMS and PMV machines

\begin{tabular}{c|c|c|c}
\hline & PMS & PMV I & PMV II \\
\hline$i_{a} \cdot R_{a}\left[\mathrm{~V}_{\mathrm{rms}}\right]$ & 5.93 & 7.85 & 4.47 \\
\hline$L_{s} \cdot d i_{a} / d t\left[\mathrm{~V}_{\mathrm{rms}}\right]$ & 29.58 & 117.01 & 57.94 \\
\hline$e_{a}\left[\mathrm{~V}_{\mathrm{rms}}\right]$ & 100.85 & 99.49 & 102.14 \\
\hline
\end{tabular}

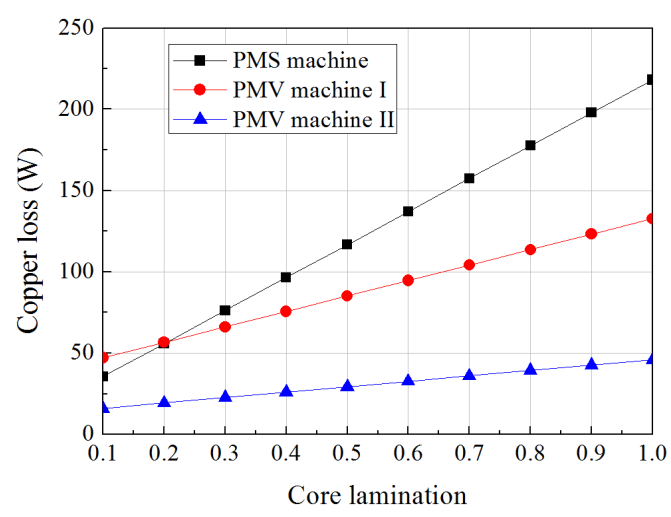

Fig. 7. Variations of the copper loss according to the core lamination

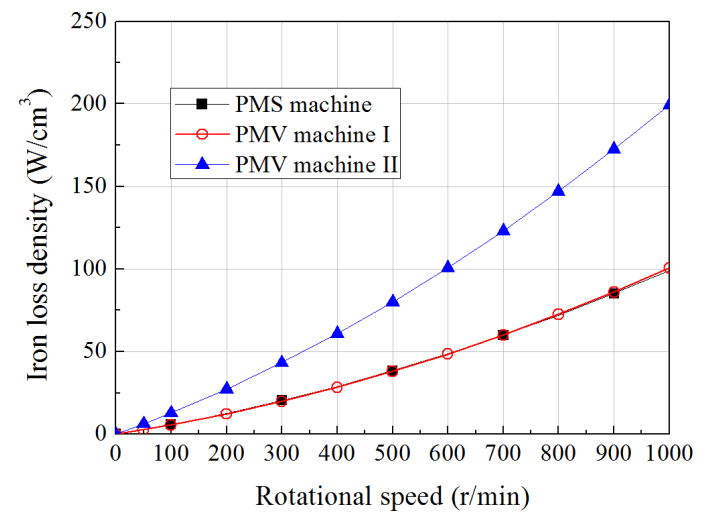

Fig. 8. Variations of the iron-loss density according to the rotational speed

machine II is improved by $1 \%$, as shown in Table 3 . The copper loss of PMV machine I is $32 \%$ higher than that of the PMS machine. This is due to the increase of the resistance in the end winding, as previously mentioned, and it thus decreases the efficiency of PMV machine I. The effects of the end winding depend on the core lamination. For the case when the input current is constant, Fig. 7 shows the variations of the copper loss according to the core lamination for the PMS and PMV machines. The core lamination is changed from 26 to $260 \mathrm{~mm}$, and the variable dimensions are expressed as a ratio of the core lamination to the outer diameter of the rotor. Although the increase in the core lamination improves the output power of the machines, the output power per unit volume is the same for each machine. Compared with the PMS machine, the copper loss of PMV machine II is lower regardless of the core lamination, but the copper loss of PMV machine I is lower when the ratio of the core lamination relative to the outer diameter of the rotor is over 0.2. In the case of the iron loss, the PMS machine and PMV machine I have similar values for the iron loss, as shown in Table 3. However, the iron loss of the PMV machine II has a value that is 2.2 times greater than that of the PMS machine. Fig. 8 shows the variation in the iron-loss density with rotational speed for the PMS and PMV machines. As the

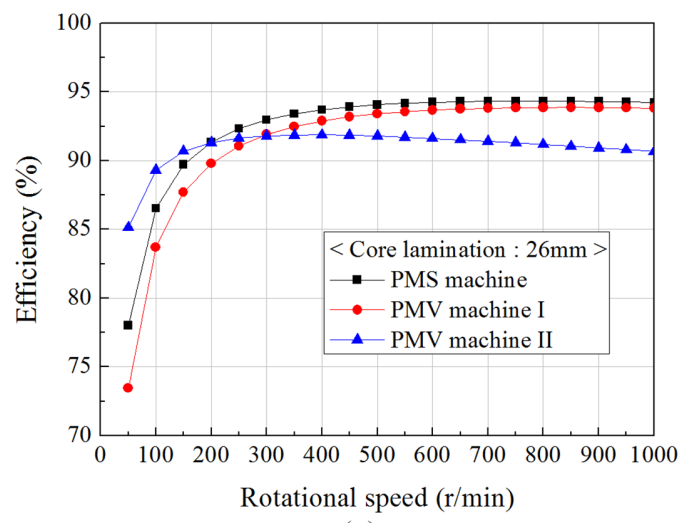

(a)

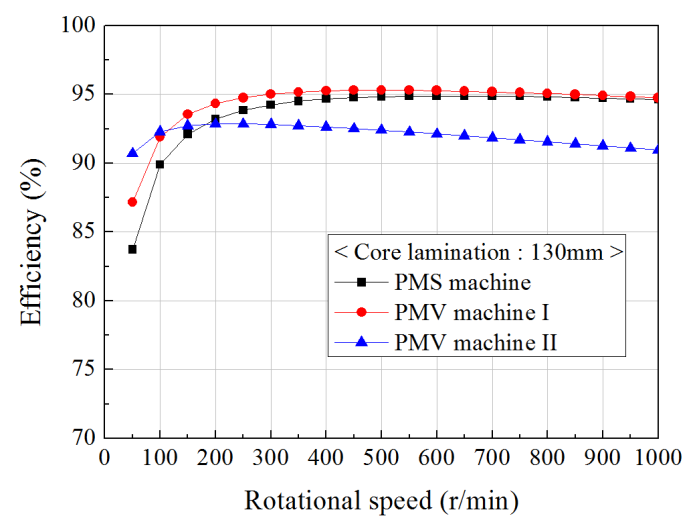

(b)

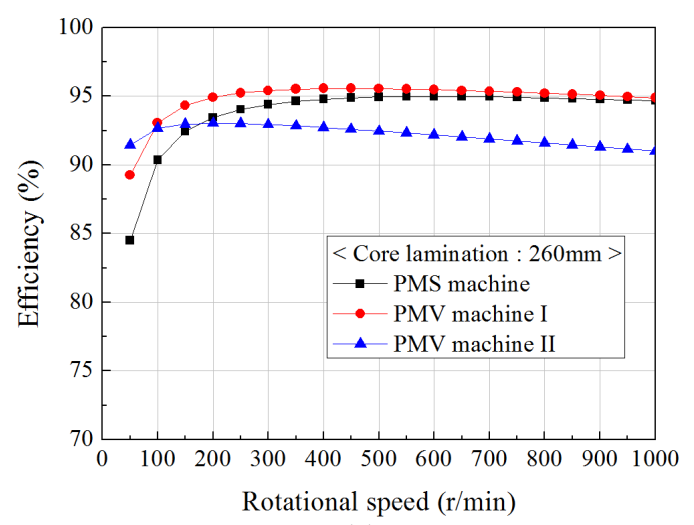

(c)

Fig. 9. Comparison between efficiency and rotational speed with variations in core laminations: (a) core lamination $26 \mathrm{~mm}$; (b) core lamination $130 \mathrm{~mm}$; and (c) core lamination $260 \mathrm{~mm}$ 
rotational speed increases, the iron-loss density of PMV machine II increases significantly compared with the PMS machine and PMV machine I. Thus, the difference in efficiency of the PMS and PMV machine II can be varied according to the rotational speed.

Fig. 9 compares the efficiency of the PMS and PMV machines when the rotational speed and the core lamination are varied under the condition of identical output power per unit volume. PMV machine I tends to have a higher efficiency than that of the PMS machine when the ratio of the core lamination to the outer diameter of the machine is over 0.2. The difference in the efficiency between the PMS machine and PMV machine I is reduced as the rotational speed increases. PMV machine II has less copper loss and higher iron loss when compared to that of the PMS machine and PMV machine I. Therefore, at low speed, the efficiency of PMV machine II has the highest value compared to that of the other machines. In addition, the difference in the efficiency of the PMS machine and PMV machine II increases with a decrease in the core lamination at low speeds. For example, when the core lamination is 26 $\mathrm{mm}$ and the rotational speed is $50 \mathrm{rpm}$, the efficiency of the PMV machines is $7 \%$ higher than that of the PMS machine, as shown in Fig. 9(a).

\section{Conclusion}

This paper compares the performances of PMS and PMV machines under the condition of the same output power per unit volume. We considered two types of PMV machines. One has surface-mounted PMs on the rotor and the other has inserted PMs between the FMPs on the stator and teeth on the rotor. Compared to the PMS machine, the magnetic gearing effect in PMV machines can reduce the number of slots and the number of turns per phase. In addition, the PMV machines used fewer PM materials than the PMS machine. However, the inductor voltages of the stator windings for the PMV machines were much higher than those of the PMS machine. This resulted in a decrease of the power factor in the PMV machines. As is well known, the low power factor of PMV machines requires the application of a power factor-correction method, which increases the cost of the control system. However, considering the amount of PM materials used, PMV machines have a comparable cost. With respect to the efficiency, PMV machine I has a better performance than the PMS machine when the ratio of the core lamination to the outer diameter of the machine is over 0.2. Further, PMV machine II has less copper loss and higher iron loss when compared to the PMS machine and PMV machine I. Thus, the efficiency of PMV machine II is superior at low rotational speeds compared to the other machines discussed in this paper.

\section{Acknowledgements}

This work was supported by the Basic Science Research Program through the National Research Foundation of Korea (NRF), and was funded by the Ministry of Education, Science, and Technology (NRF-2012R1A1A 2044307).

\section{References}

[1] A. Toba and T. A. Lipo, "Generic Torque-maximizing Design Methodology of Surface Permanent-magnet Vernier machine," IEEE Transactions on Industry Applications, vol. 36 , no. 6, pp. 1539-1546, Nov. 2000.

[2] S. Ho, S. Niu, and W. Fu, "Design and Comparison of Vernier Permanent Magnet Machines," IEEE Transactions on Magnetics, vol. 47, no. 10, pp. 32803283, Oct. 2011.

[3] A. Ishizaki, T. Tanaka, K. Takasaki, and S. Nishikata, "Theory and Optimum Design of PM Vernier Motor," in Proc. of Int. Conf. Electr. Mach. Drives, Durham, USA, no. 412, pp. 208-212, Sep. 1995.

[4] S. Niu, S. L. Ho, W. N. Fu, and L. L. Wang, "Quantitative Comparison of Novel Vernier Permanent Magnet Machines," IEEE Transactions on Magnetics, vol. 46, no. 6, pp. 2032-2035, Jun. 2010.

[5] S. U. Chung, Y. D. Chun, B. C. Woo, D. K. Hong, and J. Y. Lee, "Design Considerations and Validation of Permanent Magnet Vernier Machine with Consequent Pole Rotor for Low Speed Servo Applications," JEET, vol. 8, no. 5, pp. 1146-1151, Sep. 2013.

[6] G. Bertotti, "General Properties of Power Losses in Soft Ferromagnetic Materials," IEEE Transactions on Magnetics, vol. 24, no. 1, pp. 621-630, Jan. 1988.

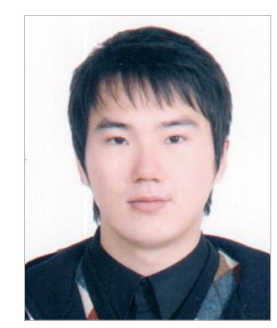

Dae-Kyu Jang He received B.S. and M.S. degree in electrical engineering from Donga University, Busan, Rep. of Korea in 2011 and 2013, respectively. From 2013 to 2014, he worked in LG Electronics as a researcher, and engaged in the developments of electric machines for air conditioner. Since 2014, he has been studying for Ph.D. degree in electrical engineering from Donga University. His research interests are design and analysis of electro-mechanical systems including driving circuits. 


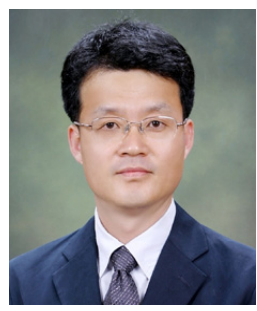

Jung-Hwan Chang He received B.S. and M.S. degrees in electrical engineering and Ph.D. degree in precision mechanical engineering from Hanyang University, Seoul, Rep. of Korea in 1994, 1997 and 2001, respectively. From 2001 to 2002, he worked at Institute of Brain Korea 21 at Hanyang University, where he developed micro drive and high-speed spindle motor. From 2002 to 2003, he worked as research fellow at University of California at Berkeley with the support of Korea Science and Engineering Foundation, and analyzed and developed electrically controlled engine valve system. From 2003 to 2009, he worked in Korea Electrotechnology Research Institute (KERI) as a senior researcher, and engaged in the developments of special purpose machines. Since 2009, he has been with the department of electrical engineering, Donga University, Busan Rep. of Korea, as an associate professor. His interests are the design and analysis of electro-mechanical systems including driving circuits. 